\title{
Six-transmembrane epithelial antigen of the prostate 1 accelerates cell proliferation by targeting c-Myc in liver cancer cells
}

\author{
KAZUTAKA IIJIMA $^{1 *}$, HAJIME NAKAMURA ${ }^{1 *}$, KOHICHI TAKADA $^{1}$, NAOTAKA HAYASAKA ${ }^{1}$, \\ TOMOHIRO KUBO $^{1}$, YUI UMEYAMA ${ }^{1}$, SATOSHI IYAMA ${ }^{2}$, KOJI MIYANISHI ${ }^{1}$, \\ MASAYOSHI KOBUNE ${ }^{2}$ and JUNJI KATO ${ }^{1}$ \\ Departments of ${ }^{1}$ Medical Oncology and ${ }^{2}$ Hematology, Sapporo Medical University \\ School of Medicine, Sapporo, Hokkaido 060-8543, Japan
}

Received March 5, 2021; Accepted May 11, 2021

DOI: $10.3892 / \mathrm{ol} .2021 .12807$

\begin{abstract}
Six-transmembrane epithelial antigen of the prostate 1 (STEAP1) has emerged as an ideal target in cancer therapeutics. However, the functions of STEAP1 in liver cancer remain unexplored. The current study aimed to characterize the biological roles of STEAP1 in liver cancer. STEAPI expression was upregulated in tumor tissues, and high STEAPI expression was associated with poor clinical outcomes in patients with liver cancer, according to several publicly available datasets. STEAPl silencing using small interfering RNA inhibited cell proliferation and was accompanied by $\mathrm{G}_{1}$ arrest induced by the suppression of cyclin D1 and the promotion of p27. STEAP1 silencing suppressed c-Myc expression, which was identified as a component in STEAP1 signal transduction by mining publicly available datasets and was then confirmed by PCR array. In conclusion, the knockdown of STEAPl in liver cancer cell lines led to inhibition of cell proliferation involving $\mathrm{G}_{1}$ arrest by suppressing c-Myc. The present study provides a preclinical concept for STEAPI as a druggable target in liver cancer.
\end{abstract}

\section{Introduction}

Primary liver cancer is estimated to be the third leading cause of cancer-related deaths worldwide, accounting for 830,000 deaths each year (1). Hepatocellular carcinoma (HCC) is the most common type of primary liver cancer comprising $75-85 \%$ of cases (1). Despite recent advances in multikinase

Correspondence to: Dr Kohichi Takada, Department of Medical Oncology, Sapporo Medical University School of Medicine, South-1, West-16, Chuo, Sapporo, Hokkaido 060-8543, Japan

E-mail: author@mail.com

*Contributed equally

Key words: six-transmembrane epithelial antigen of the prostate 1, c-Myc, G1 arrest, liver cancer, hepatocellular carcinoma, cancer therapeutics inhibitors, such as sorafenib, regorafenib, and lenvatinib, as well as anti-vascular endothelial growth factor therapies and immune check point inhibitors, advanced HCC has a dismal prognosis (2-5). An exploration of the molecular characteristics of liver cancer is needed to develop more effective therapeutics.

A well characterized oncoprotein, c-Myc contributes to the pathogenesis of a broad range of human cancers, including liver cancer (6). Overexpression of c-Myc is associated with a poor prognosis (7). The amplification of $c-M y c$ and alterations of proximal c-Myc network members have been identified in over $30 \%$ and $70 \%$ of HCC cases, respectively (8). Such findings highlight c-Myc as an attractive target for liver cancer therapeutics. However, its structure, which lacks a druggable hydrophobic pocket, and its nuclear localization have hampered the development of specific inhibitors of c-Myc (9). Certainly, ongoing clinical trials of c-Myc inhibitors are non-existent, except for a trial involving 90-amino acid peptide as a dominant negative inhibitor (10). Specifically, it is critical to seek druggable targets in the c-Myc pathway to combat c-Myc-driven liver cancer.

Six-transmembrane epithelial antigen of the prostate 1 (STEAP1), which was initially identified in prostate cancer cells and is expressed at low levels in normal cells, is a cell surface protein (11) that is over-expressed in many human cancers (12). STEAP1 has thus emerged as an ideal target in cancer therapeutics. STEAP1 is believed to play a physiological role as an ion channel and transporter (13). Additionally, structural analyses using a cryo-electron microscopy revealed that STEAP1 works as a ferric reductase when binding to the NADPH-binding domain of STEAP4 (14). In contrast, the pathological functions of STEAP1 in cancer cells have been largely unexplored. We recently discovered that high expression of STEAP1 lead to the suppression of reactive oxygen species (ROS) that escaped from apoptosis via a NF-E2-related factor 2 (NRF2) pathway in colorectal cancer cells (15). However, the roles of STEAP1 in liver cancer pathogenesis remain completely unknown.

Here, we sought to characterize the biological roles of STEAP1 in liver cancer. We identified that STEAPI transcript levels were significantly increased in liver cancer compared to normal liver cells, and that such high levels were associ- 
ated with a poor prognosis. The knockdown of STEAPI led to cell-growth inhibition accompanied by $\mathrm{G}_{1}$ arrest by targeting the suppression of c-Myc, which was discovered by mining publicly available databases. Our findings yield a new treatment strategy targeting the STEAP1-c-Myc axis in liver cancer.

\section{Materials and methods}

Databases and gene expression data analysis. Gene expression levels of STEAP1 in non-tumor and liver cancer tissues were evaluated using gene expression profiles of GSE14520 and GSE36376 from the Gene Expression Omnibus, a public and freely available database. The GSE14520 dataset includes 488 samples of 241 non-cancerous and 247 cancerous hepatic tissues. These datasets have been widely used and well accepted in bioinformatics analysis of liver cancer. The GSE36376 dataset includes 433 samples consisting of 193 non-cancerous hepatic tissues and 240 cancerous tissues. The correlation between STEAP1 levels and clinical outcomes of patients with liver cancer was investigated using GSE14520 and the Cancer Genome Atlas Program (TCGA) (16). We used a receiver operating characteristic curve to determine the cutoff value. In total, 247 patients from the GSE14520 dataset and 360 patients from TCGA, all with liver cancer, were divided into two groups having high or low levels of STEAP1, respectively. Kaplan-Meier analyses of survival were performed based on these groups. Statistical analyses were performed using EZR software version 1.33 (17).

Gene set enrichment analysis (GSEA) was performed using the open source software, GSEA 4.0.3. Initially, we set two groups (STEAP1_high and STEAP1_low) in GSE14520-GPL3921, which includes 225 liver cancer samples in total. We conducted GSEA of the two groups using Hallmark gene sets. Gene sets showing a NOM P-val. (P-value) $<0.05$ and false discovery rate (FDR) Q-val. (FDR) $<0.25$ were considered significant. Differentially expressed genes (DEGs) between these two groups were identified using an online tool, GEO2R, with $\mid \log \mathrm{FCl}>1.5$ and an adjusted $\mathrm{P}$-value $<0.05$.

Cell lines and culture conditions. HepG2 and Hep3B cell lines were purchased from the American Type Culture Collection; these were authenticated by short tandem repeat DNA profiling prior to all experiments. Both cell lines were cultured in DMEM containing 10\% fetal bovine serum (FBS), $2 \mu \mathrm{M}$ L-glutamine and $1 \%$ penicillin-streptomycin (the medium and all supplements from Sigma-Aldrich; Merck KGaA).

Inhibition of STEAPl expression by small-interfering RNA. Control small-interfering RNA (siRNA; Control; \#4390843; Thermo Fisher Scientific, Inc.) and two independent siRNAs targeting human STEAP1 (siSTEAP1; D-003713-01: 5'-GGA GAGAAUUUCACUAUAU-3' and D-003713-02: 5'-UAAAGA AGAUGCCUGGAUU-3'; Dharmacon) were transfected using Lipofectamine RNAiMAX (Thermo Fisher Scientific, Inc.) according to the manufacturer's protocol. Cells were seeded at a density of $3 \times 10^{5}$ cells/well into 6-well plates and cultured for $24 \mathrm{~h}$ at $37^{\circ} \mathrm{C}$. Subsequently, cells were transfected with control siRNA or siRNA targeting human STEAPl, and incubated for $72 \mathrm{~h}$ at $37^{\circ} \mathrm{C}$. Final siRNA used per well was 25 pmol. After incubation, floating cells in media were collected, adhesive cells were washed and collected, and both were immediately used for experiments.

Reverse transcription-quantitative PCR (RT-qPCR). Total RNA was extracted using TRIzol Reagent (Thermo Fisher Scientific) according to the manufacturer's protocol. Subsequently, complementary (c)DNA was synthesized from the RNA using a SuperScript VILO cDNA synthesis kit (Thermo Fisher Scientific). qPCR was performed with an Applied Biosystems 7300 Real-time PCR system (Applied Biosystems; Thermo Fisher Scientific, Inc.). The analysis of target genes (STEAPI and $c-M y c$ ) was conducted in quadruplicate using a POWER SYBR-Green Master Mix (Thermo Fisher Scientific, Inc.) as previously described (18). The thermal profile of the qPCR program consisted of $2 \mathrm{~min}$ at $50^{\circ} \mathrm{C}, 10 \mathrm{~min}$ at $95^{\circ} \mathrm{C}$, 40 cycles of $15 \mathrm{sec}$ at $95^{\circ} \mathrm{C}$ and $1 \mathrm{~min}$ at $60^{\circ} \mathrm{C}$, and a dissociation stage at the end of the run from $60^{\circ} \mathrm{C}$ to $95^{\circ} \mathrm{C}$. Transcript levels were normalized to $\beta$-actin expression and analyzed using the $2^{-\Delta \Delta \mathrm{Cq}}$ method. The following PCR primers were designed: 5'-CCCTTCTACTGGGCACAATACA-3' and 5'-GCATGG CAGGAATAGTATGCTTT-3' for STEAPI; 5'-TTTTTCGGG TAGTGGAAAACC-3' and 5'-GCAGTAGAAATACGGCTG CAC-3' for $c$-Myc; and 5'-GGCATCCTCACCCTGAAGTA-3' and 5'-GAAGGTGTGGTGCCAGATTT-3' for $\beta$-actin .

Western blotting. As previously described (19), cells were solubilized in radioimmunoprecipitation assay lysis buffer $(50 \mathrm{mM}$ Tris- $\mathrm{HCl}, \mathrm{pH} 7.5,1 \%$ NP-40, 0.5\% Na-deoxycholate, $1 \mathrm{mM}$ EDTA, $150 \mathrm{mM} \mathrm{NaCl}, 1 \mathrm{mM}$ EGTA, and protease inhibitor cocktail; Sigma-Aldrich; Merck KGaA), and centrifuged at $12,000 \times \mathrm{g}$ for $10 \mathrm{~min}$. The supernatants were collected, and protein concentrations were determined using a bicinchoninic acid Protein Assay Kit (Thermo Fisher Scientific, Inc.). Equal amounts of protein were separated on MULTIGEL II mini gels (Cosmo Bio Co., Ltd.) and transferred to polyvinylidene fluoride membranes using a QBlot Kit (ATTO, Tokyo, Japan). The blots were probed using the following primary antibodies: anti-STEAP1 (sc25514; Santa Cruz Biotechnology), anti-STEAP1 (\#88677; Cell Signaling Technology), anti-cyclin D1 (\#2987; Cell Signaling Technology,), anti-p27 Kip1 (\#3686; Cell Signaling Technology), anti-c-Myc (OP10L; EMD Biosciences), and anti-actin-horse radish peroxidase (HRP; sc-47778; Santa Cruz Biotechnology).

Evaluation of cell proliferation. Hepatocellular carcinoma cells were seeded at a density of $2 \times 10^{3}$ cells/well into 96 -well plates. Control siRNA or two independent siRNAs targeting human STEAPl were transfected $24 \mathrm{~h}$ after seeding. Cell viability was assessed at 0, 24, 48 and $72 \mathrm{~h}$ using a WST-1 assay (Premix WST-a Cell Proliferation Assay; Takara Bio) and Infinite M1000 Pro microplate reader (Tecan Japan). A growth curve was constructed by plotting absorbance against time.

Cell cycle analysis. Liver cancer cells were seeded at a density of $3 \times 10^{5}$ cells/well into 6 -well plates and cultured for $24 \mathrm{~h}$. Subsequently, cells were transfected with control siRNA or an siRNA targeting human STEAPl, and incubated for $72 \mathrm{~h}$. After incubation, floating cells in media were collected and adhesive 
A

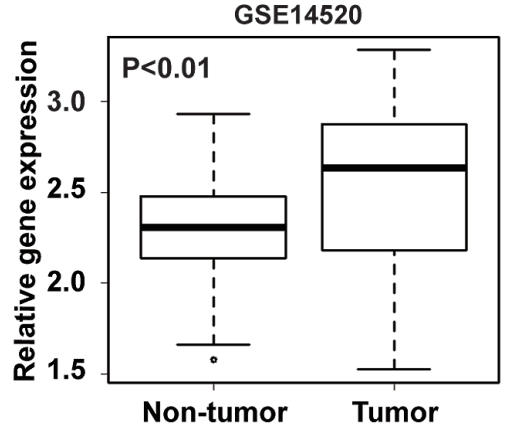

B

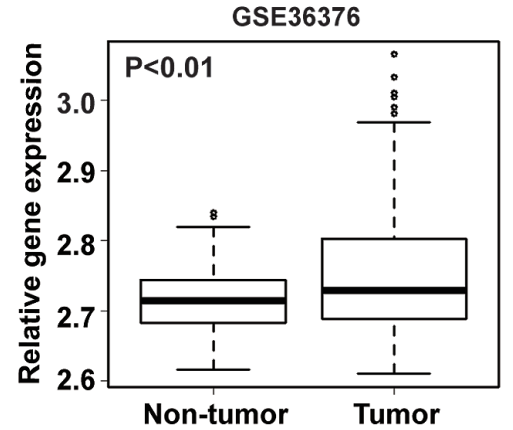

C

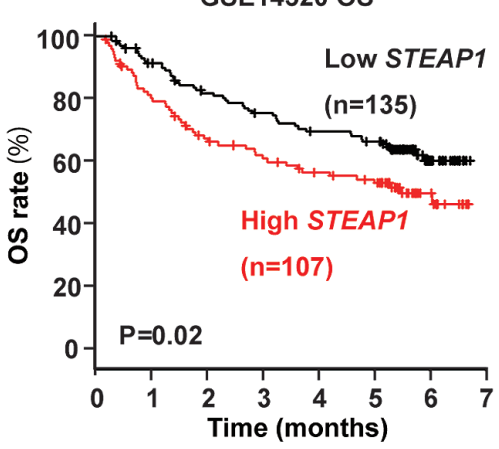

D

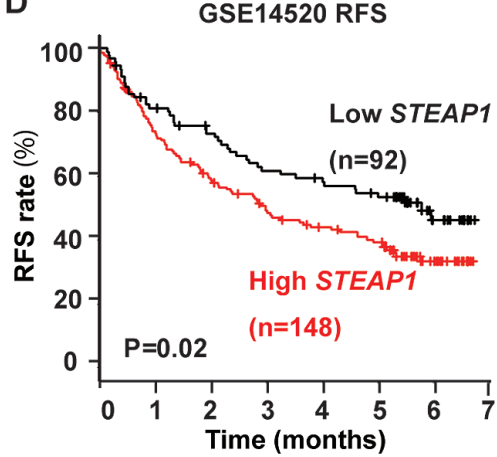

E

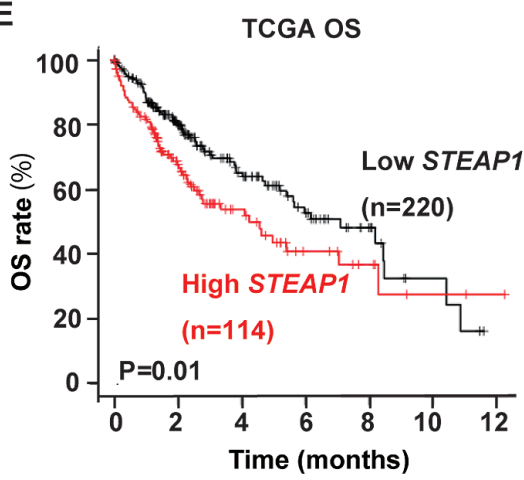

Figure 1. STEAP1 expression is upregulated and associated with poor survival in patients with liver cancer. Publicly accessible gene expression profiling datasets, (A) GSE14520 and (B) GSE36376, were obtained from the Gene Expression Omnibus and analyzed to evaluate STEAP1 expression in patients with liver cancer. Associations between STEAP1 expression and (C) OS and (D) RFS in GSE14520, and (E) OS in TCGA were evaluated using the Kaplan-Meier method. STEAP1, six-transmembrane epithelial antigen of the prostate 1; OS, overall survival; RFS, recurrence-free survival; TCGA, The Cancer Genome Atlas.

cells were washed, fixed in ethanol, and stained with propidium iodide using a cell-cycle analysis kit (FxCycle PI/RNase Staining Solution; Thermo Fisher Scientific), followed by analysis on a BD FACS II (BD Biosciences) instrument using FACSDiva (BD Biosciences) as previously described (20).

Apoptosis assay. Apoptosis was evaluated using an Annexin V/7-amino-actinomycin (AAD) staining kit (BD Biosciences). Liver cancer cells were seeded at a density of $3 \times 10^{5}$ cells/well into 6-well plates and cultured for $24 \mathrm{~h}$. Subsequently, cells were transfected with control siRNA or an siRNA targeting human STEAP1, and incubated for $72 \mathrm{~h}$. After incubation, floating cells in media were collected and adhesive cells were washed, stained with Annexin V and 7-AAD, and analyzed on a BD FACSCanto II (BD Biosciences) instrument using FACSDiva (BD Biosciences) as previously described (21).

PCR array. Total RNA was reverse-transcribed using an $\mathrm{RT}^{2}$ First Strand Kit (Qiagen). PCR array was performed using RT ${ }^{2}$ Profiler $^{\mathrm{TM}}$ PCR Array Human MYC Targets (PAHS-177Z; Qiagen) according to the manufacturer's protocol.

Statistical analysis. The significance of differences was determined by Student's t-test, Mann-Whitney U test, log-rank test or one-way ANOVA followed by Bonferroni's post-hoc test, as appropriate. Pearson's correlation was used to perform the correlation analysis. All statistical analyses were performed using EZR software version 1.33 (17). Statistical significance was defined as $\mathrm{P}<0.05$.

\section{Results}

STEAP1 is up-regulated and significantly associated with poor overall survival and recurrence-free survival in liver cancer. We first investigated the expression of STEAPI in patients with liver cancer using publicly accessible datasets (GSE14250 and GSE36376) from the Gene Expression Omnibus. In both datasets, STEAPl is over-expressed in liver cancer tissues compared to non-cancerous hepatic tissues (Fig. 1A and B). Next, we evaluated the correlation between STEAPl expression and survival in patients with liver cancer using GSE14520 and TCGA datasets. Patients with high STEAPI expression presented with significantly shorter overall survival (OS) and recurrence-free survival (RFS) in GSE14520 and significantly shorter OS in TCGA (Fig. 1C-E). These data imply that STEAP1 may have oncogenic functions in liver cancer.

Knockdown of STEAP1 inhibits proliferation of liver cancer cell lines. To evaluate the effect of STEAP1 on liver cancer, we performed STEAPl silencing using an RNA interference method in two different liver cancer cell lines, HepG2 and Hep3B. Knockdown efficiency was examined by RT-qPCR and western blot. STEAP1 expression in these cell lines was significantly down-regulated $72 \mathrm{~h}$ after transfection of two independent siRNAs (Fig. 2A, B, D and E). We next evaluated the impact of STEAPI silencing on liver cancer cell lines using WST-1 assays. STEAP1 silencing significantly reduced proliferation in both cell lines (Fig. 2C and F). Based on these data, we concluded that STEAP1 activated proliferation in liver cancer cell lines. 
A

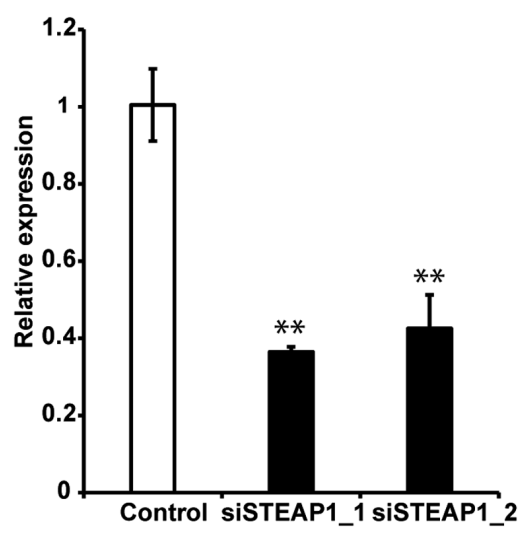

C

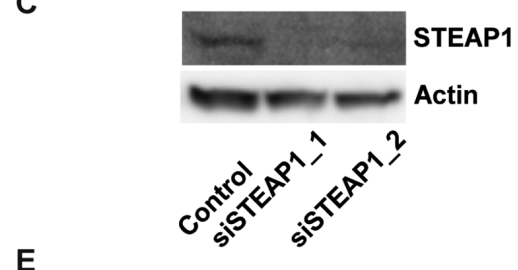

E

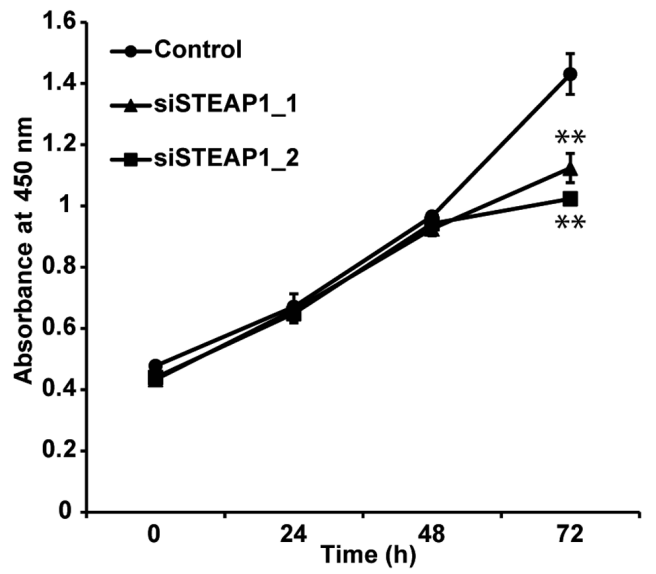

B

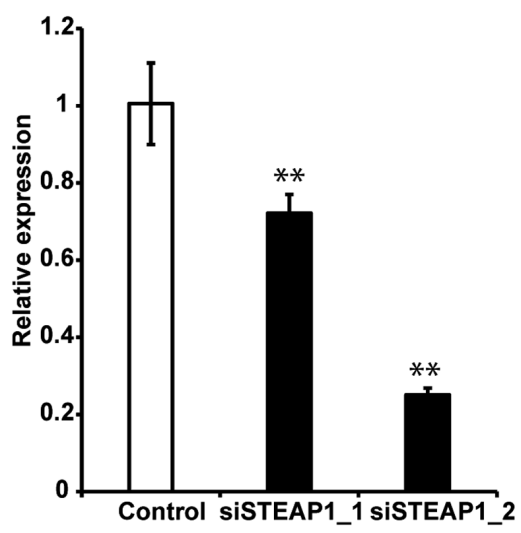

D

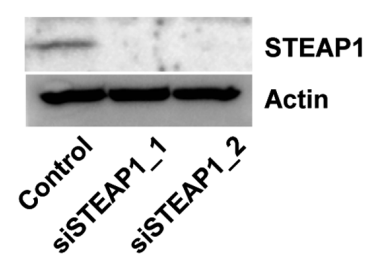

F

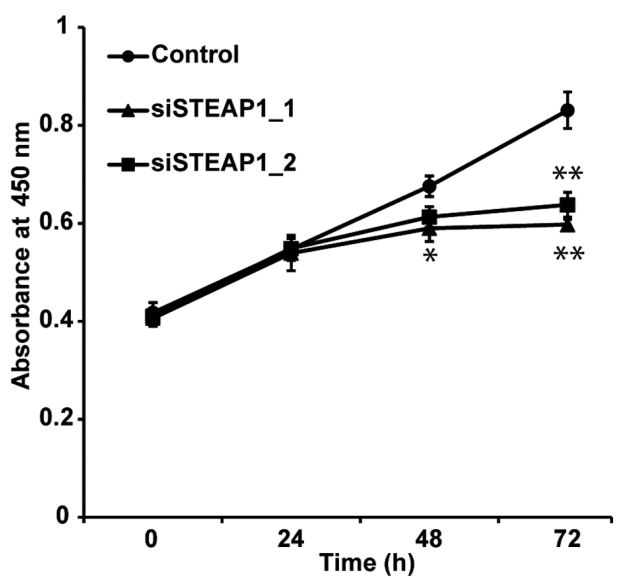

Figure 2. Knockdown of STEAP1 leads to inhibition of cell proliferation in different liver cancer cell lines. Two different liver cancer cell lines, HepG2 and Hep3B, were transfected with non-targeting control siRNA and two independent siRNAs targeting (siSTEAP1_1 and siSTEAP1_2). Efficiency of knockdown was evaluated using RT-qPCR and western blotting. RT-qPCR in (A) HepG2 and (B) Hep3B cells. Western blotting in (C) HepG2 and (D) Hep3B. Data of RT-qPCR experiments are shown as the mean of quadruplicate measurements \pm SD. Cell proliferation of two different liver cancer cell lines, (E) HepG2 and (F) Hep3B, was evaluated using a WST-1 assay at 0,24,48 and $96 \mathrm{~h}$ after siRNA transfection. Data are presented as the mean of triplicate measurements $\pm \mathrm{SD}$. ${ }^{*} \mathrm{P}<0.05$ and ${ }^{* * *} \mathrm{P}<0.01$ vs. control. RT-qPCR, reverse transcription-quantitative PCR; siRNA, small interfering RNA; STEAP1, six-transmembrane epithelial antigen of the prostate 1.

STEAP1 silencing promotes $G_{1}$ arrest in liver cancer cell lines. To evaluate the mechanism of decreasing proliferation in response to the knockdown of STEAPI, we examined the effects of STEAP1 silencing on the cell cycle in the liver cancer cell lines, HepG2 and Hep3B. STEAP1 silencing significantly induced $\mathrm{G}_{1}$ arrest in both liver cancer cell lines (Fig. 3A and B). We also performed a flow cytometry analysis using Annexin V/7AAD staining to evaluate the rate of apoptosis. However, an increased percentage of apoptosis was not observed in STEAPl-silenced liver cancer cell lines (Fig. S1A and B). To analyze the mechanism of $\mathrm{G}_{1}$ arrest in HCC cell lines induced by the knockdown of STEAP1, we evaluated protein levels of several cell-cycle-related proteins in liver cancer cell lines using western blot. The expression of the $\mathrm{G}_{1}$ arrest-associated protein, cyclin D1, was decreased, whereas the expression of $\mathrm{p} 27$, which promotes cell-cycle arrest, was apparently increased (Fig. 3C).
c-Myc target genes were significantly enriched in patients with liver cancer showing high STEAPl expression. To clarify the pathways related to STEAP1, we first extracted DEGs between low and high STEAPl liver cancer samples in a publicly accessible dataset, GSE14520-GPL3921, using GEO2R. The significant DEGs with $\mid \log \mathrm{FCl}>1.5$ and adjusted $\mathrm{P}$-value $<0.05$ are highlighted in red and blue colors. Each gene was represented as a volcano plot (Fig. 4A) and listed in a table (Table I). Next, we conducted GSEA to explore the gene sets regulated by STEAP1 in liver cancer and found five pathways which were significantly enriched (NOM P-val $<0.05$ and FDR Q-val <0.25; Fig. 4B, Fig. S2, and Table SI). The genes belonging to MYC_TARGET_V2 were the most significantly enriched among these five pathways (Fig. 4C and D). Based on these findings, we hypothesized the existence of a relationship between STEAP1 and c-Myc in liver cancer. To confirm this, we evaluated their expression using the publicly accessible data- 
Table I. List of significant DEGs in samples with high and low STEAPl expression in publicly accessible gene expression profiling dataset, GSE14520-GPL3921.

A, Upregulated DEGs

\begin{tabular}{|c|c|c|c|}
\hline Symbol & Gene name & $\log _{2}$ ratio & Adjusted P-value \\
\hline AFP & $\alpha$ fetoprotein & 2.28 & 0.0197 \\
\hline SULT1C2 & Sulfotransferase family $1 \mathrm{C}$ member 2 & 2.23 & 0.0000113 \\
\hline MT1E & Metallothionein 1E & 2.09 & 0.0000135 \\
\hline $\mathrm{ABCB} 1$ & ATP binding cassette subfamily B member 1 & 1.99 & 0.0000268 \\
\hline MT1G & Metallothionein $1 \mathrm{G}$ & 1.96 & 0.0000135 \\
\hline GPX2 & Glutathione peroxidase 2 & 1.92 & 0.00308 \\
\hline C9 & Complement component 9 & 1.92 & 0.00971 \\
\hline MT1H & Metallothionein $1 \mathrm{H}$ & 1.91 & 0.0000105 \\
\hline SPP1 & Secreted phosphoprotein 1 & 1.91 & 0.0104 \\
\hline MT1X & Metallothionein $1 \mathrm{X}$ & 1.86 & 0.0000241 \\
\hline REG3A & Regenerating family member $3 \alpha$ & 1.83 & 0.0215 \\
\hline ROBO1 & Roundabout guidance receptor 1 & 1.82 & 0.0000441 \\
\hline LCN2 & Lipocalin 2 & 1.8 & 0.00455 \\
\hline MYC & v-myc avian myelocytomatosis viral oncogene homolog & 1.74 & 0.00023 \\
\hline MT1M & Metallothionein 1M & 1.71 & 0.000015 \\
\hline TSPAN8 & Tetraspanin 8 & 1.67 & 0.00928 \\
\hline PLPPR1 & Phospholipid phosphatase related 1 & 1.64 & 0.00000564 \\
\hline MT1X & Metallothionein $1 \mathrm{X}$ & 1.64 & 0.000126 \\
\hline MT1F & Metallothionein $1 \mathrm{~F}$ & 1.63 & 0.0000604 \\
\hline BCHE & Butyrylcholinesterase & 1.61 & 0.0103 \\
\hline MT1HL1 & Metallothionein 1H-like 1 & 1.6 & 0.0000192 \\
\hline MTTP & Microsomal triglyceride transfer protein & 1.6 & 0.000745 \\
\hline SQSTM1 & Sequestosome 1 & 1.59 & 0.000114 \\
\hline RELN & Reelin & 1.59 & 0.0144 \\
\hline CXCL5 & $\mathrm{C}-\mathrm{X}-\mathrm{C}$ motif chemokine ligand 5 & 1.57 & 0.000184 \\
\hline TRIM16L///TRIM16 & $\begin{array}{l}\text { Tripartite motif containing } 16 \text {-like///tripartite } \\
\text { motif containing } 16\end{array}$ & 1.57 & 0.000923 \\
\hline AKR1C4 & Aldo-keto reductase family 1, member $\mathrm{C} 4$ & 1.57 & 0.00464 \\
\hline CCL20 & C-C motif chemokine ligand 20 & 1.56 & 0.00949 \\
\hline COL2A1 & Collagen type II $\alpha 1$ chain & 1.55 & 0.0134 \\
\hline YBX3 & Y-box binding protein 3 & 1.54 & 0.0000268 \\
\hline IGF2BP3 & Insulin like growth factor 2 mRNA binding protein 3 & 1.54 & 0.00289 \\
\hline
\end{tabular}

B, Downregulated DEGs

\begin{tabular}{|c|c|c|c|}
\hline Symbol & Gene name & $\log _{2}$ ratio & Adjusted P-value \\
\hline SLPI & Secretory leukocyte peptidase inhibitor & -3.17 & $3.31 \times 10^{-08}$ \\
\hline GNMT & Glycine N-methyltransferase & -1.88 & 0.0016 \\
\hline SPP2 & Secreted phosphoprotein 2 & -1.82 & 0.00581 \\
\hline LGALS4 & Galectin 4 & -1.79 & 0.0169 \\
\hline CYP7A1 & Cytochrome P450 family 7 subfamily A member 1 & -1.55 & 0.0472 \\
\hline SLC22A1 & Solute carrier family 22 member 1 & -1.55 & 0.03 \\
\hline PPP1R1A & Protein phosphatase 1 regulatory inhibitor subunit 1A & -1.53 & 0.00516 \\
\hline CHI3L1 & Chitinase 3 like 1 & -1.52 & 0.12 \\
\hline
\end{tabular}

DEGs, differentially expressed genes; STEAP1, six-transmembrane epithelial antigen of the prostate 1. 
A

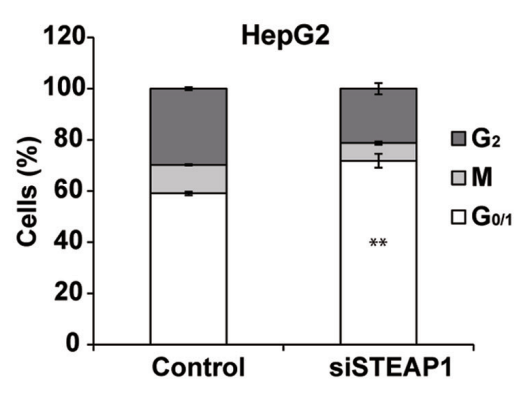

B

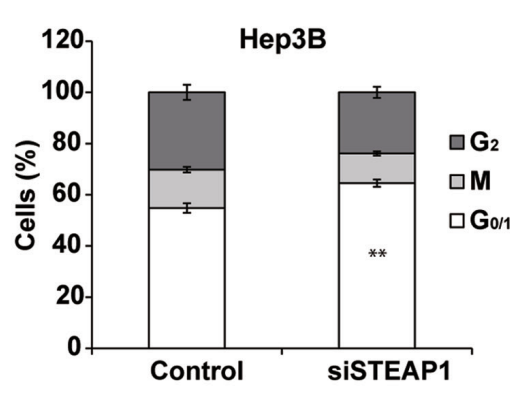

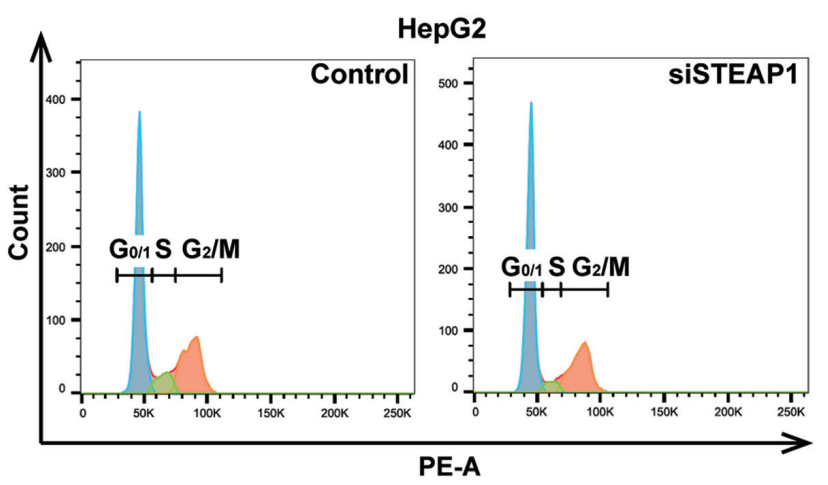

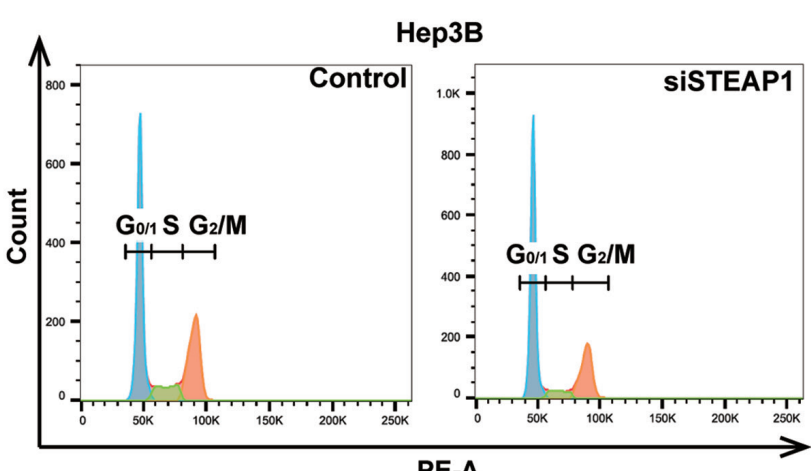

c

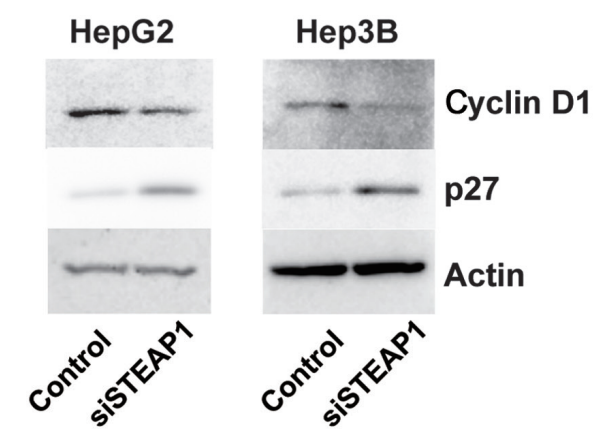

Figure 3. STEAP1 silencing triggers $\mathrm{G}_{1}$ arrest in liver cancer cell lines. Two different liver cancer cell lines, (A) HepG2 and (B) Hep3B, were transfected with non-targeting control siRNA or siSTEAP1. Cells were stained with propidium iodide $72 \mathrm{~h}$ after siRNA transfection. Subsequently, cell cycles were analyzed by flow cytometry. The percentage of $\mathrm{G}_{0 / 1}$ cells transfected with siSTEAP1 was compared to that of cells transfected with non-targeting control siRNA. Data are presented as the mean of triplicate measurements \pm SD. ${ }^{* *} \mathrm{P}<0.01$. (C) Two different liver cancer cell lines, HepG2 and Hep3B, were transfected with non-targeting control siRNA or siSTEAP1. Cell cycle-associated proteins were analyzed by western blotting. siRNA, small interfering RNA; STEAP1, six-transmembrane epithelial antigen of the prostate 1 .

sets, GSE14250, GSE36376, and TCGA. Pearson's correlation coefficient analysis revealed a significant positive relationship between STEAPl and $c-M y c$ in all datasets (Fig. 4E-G).

STEAP1 regulates $c-M y c$ and its related genes in liver cancer cell lines. To confirm the relationship between STEAP1 and $\mathrm{c}-\mathrm{Myc}$ in liver cancer, we evaluated the expression of c-Myc after STEAP1 knockdown in HepG2 and Hep3B cell lines by RT-qPCR and western blot. As we expected, downregulation of c-Myc was observed in both cell lines when transfected with siRNA targeting STEAPl compared to non-targeting siRNA (Fig. 5A-D). Next, we conducted a PCR array to analyze components of c-Myc-related genes; most were significantly downregulated by STEAP1 silencing (Figs. 5E and S3). Taken together, our data suggest that c-Myc lies downstream of STEAP1, and that the STEAP1-c-Myc pathway promotes cell proliferation and cell-cycle progression in liver cancer.

\section{Discussion}

Recently, treatment options for HCC have been expanding as new drugs are approved (2-5). However, unresectable HCC is an incurable disease; its median overall survival remains around a year (22). Thus, the further exploration of novel molecularly-based therapies is required to improve survival in patients with advanced HCC. c-Myc is a high priority target of liver cancer therapeutics because its pathological functions exist in a subset of liver cancer cases. The structure of c-Myc has hampered the development of c-Myc-specific inhibitors and highlights the need for further investigations of novel c-Myc signaling components as potential targets for liver cancer therapeutics. The current study elucidated STEAP1 as a member of the c-Myc signal transduction pathway using in vitro and bioinformatic analyses. Inhibition of STEAPl led to the suppression of cell growth accompanied by $\mathrm{G}_{1}$ arrest in liver cancer, encour- 
A

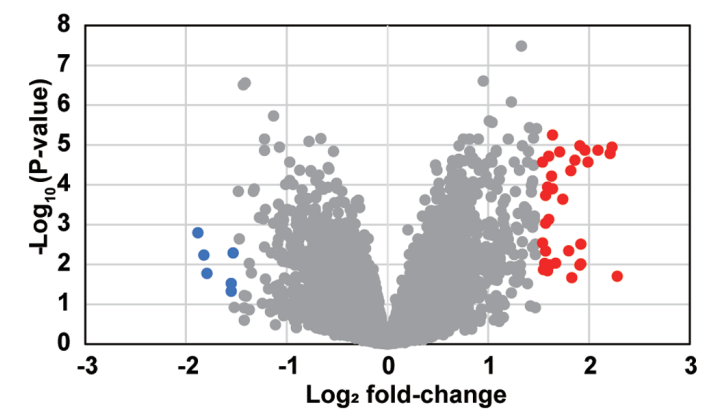

B

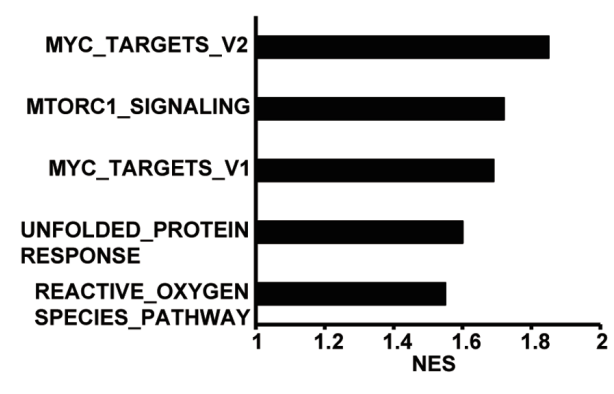

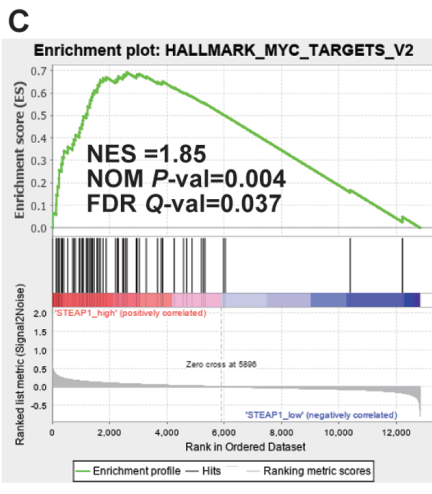

D

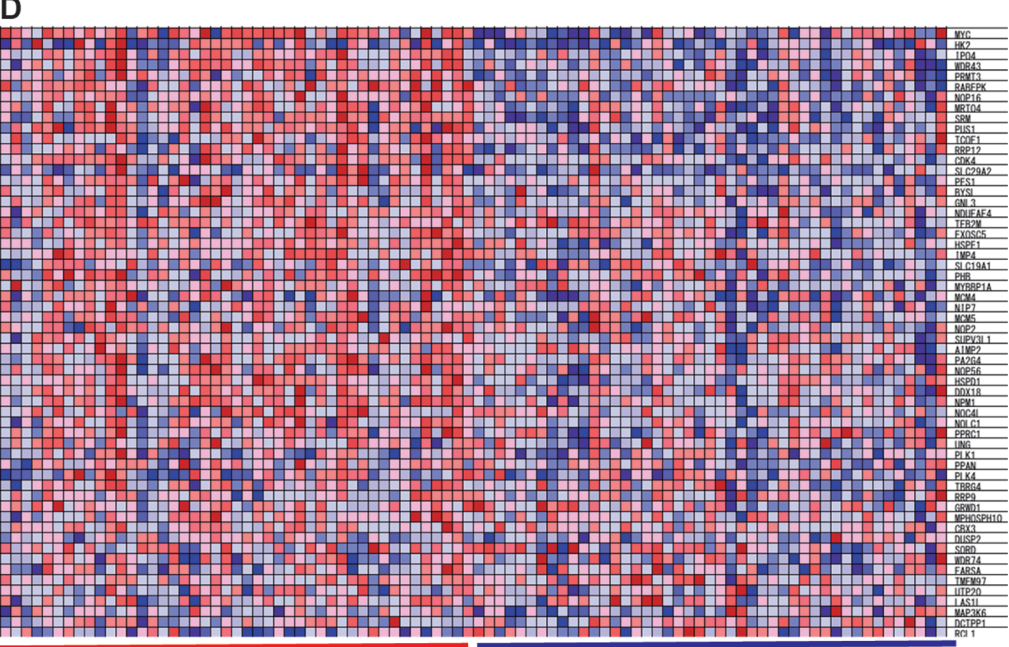

High STEAP1

E

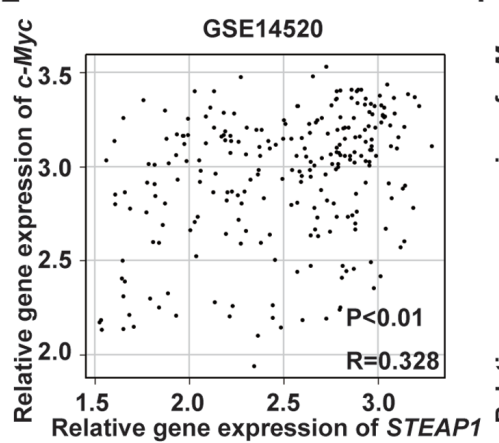

F

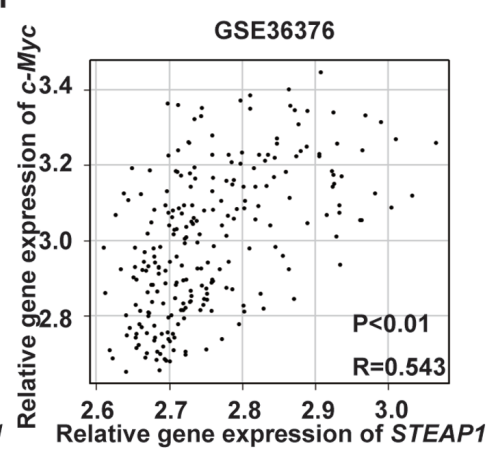

Low STEAP1

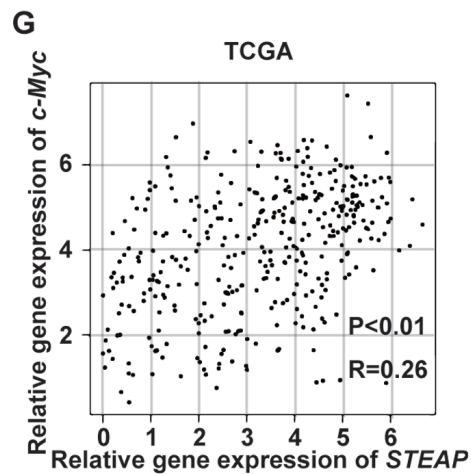

Figure 4. $c-M y c$ target genes are significantly enriched in patients with liver cancer with high STEAPl expression. (A) Volcano plot of DEGs in samples with high and low STEAP1 expression in a publicly accessible gene expression profiling dataset, GSE14520-GPL3921. Significant DEGs were defined as P $<0.05$ and a llog fold-changel $>1.5$. Red indicates upregulated genes, blue indicates downregulated genes and gray indicates non-DEGs. (B) Gene Set Enrichment Analysis was performed to explore the gene sets regulated by STEAPI in liver cancer. Bar graph showing significantly enriched Hallmark gene sets in patients with liver cancer with high STEAP1 expression. (C) Enrichment plot presentation of MYC_TARGETS_V2. (D) Heat map presentation of genes included in MYC_TARGET_V2 between samples with high and low STEAP1 expression. Correlation between STEAP1 and $c$-Myc in samples with liver cancer using publicly accessible gene expression profiling datasets: (E) GSE14520, (F) GSE36376 and (G) TCGA. DEGs, differentially expressed genes; NES, normalized enrichment score; NOM P-val, nominal P-value; FDR Q-val, false discovery rate Q-value; STEAP1, six-transmembrane epithelial antigen of the prostate 1.

aging the development of STEAP1 inhibitors as therapeutics for STEAP1-c-Myc axis-driven liver cancer. Additionally, STEAP1 is an attractive target for antibody drug conjugates (ADC) in cancers because it is expressed on the plasma membrane (11). In fact, DSTP3086S, an ADC-targeting STEAP1, has been introduced for patients with metastatic castration-resistant prostate cancer; it has been evaluated as safe and shows promising therapeutics (23). Therefore, an ADC-targeting STEAP1 can be used for patients with liver cancer, who, according to our data, show the overexpression of STEAP1 in cancerous hepatic tissue compared to adjacent non-cancerous parts (Fig. 1A and B).
In our previous work, we demonstrated that STEAPI knockdown led to apoptosis in colorectal cancer cells in an NRF2-dependent fashion, corresponding to the increased production of ROS (15). As shown in Fig. S4, intracellular ROS levels were increased by STEAPI inhibition as found in our previous work (Fig. S4A and B). Furthermore, GSEA revealed an ROS-related pathway was significantly enriched in patients with liver cancer showing upregulated STEAPl (Fig. S2D). However, as mentioned above, apoptotic cells were not increased by STEAP1 inhibition in liver cancer cells (Fig. S1A and B). In addition, we found no statistical correlation between STEAPI 
A

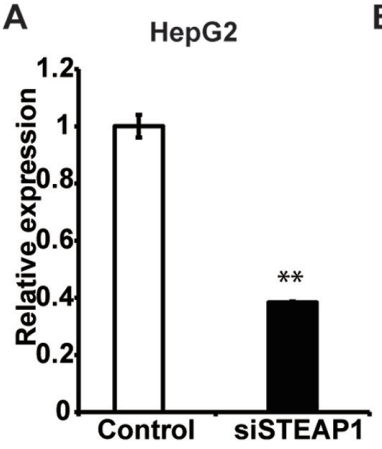

B

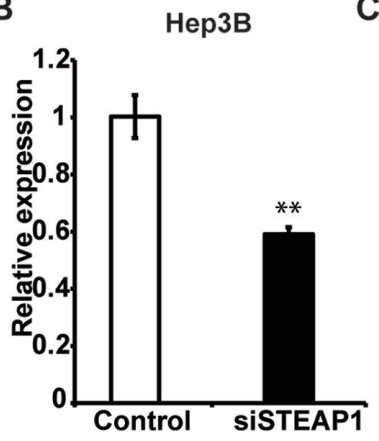

C

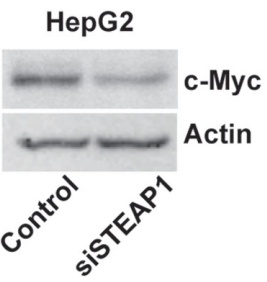

D

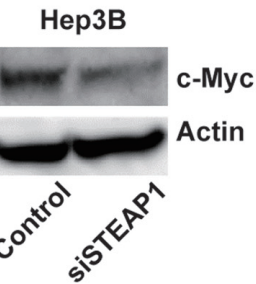

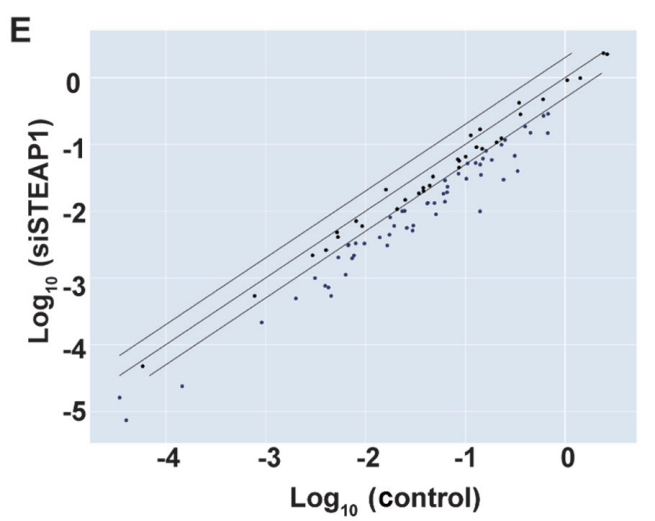

Figure 5. Inhibition of STEAP1 suppresses c-Myc and its downstream target genes in liver cancer cell lines. Two different liver cancer cell lines (HepG2 and Hep3B) were transfected with non-targeting control siRNA and siSTEAP1. c-Myc expression was evaluated by RT-qPCR and western blotting. RT-qPCR in (A) HepG2 and (B) Hep3B cells. Western blotting in (C) HepG2 and (D) Hep3B cells. Data of RT-qPCR experiments are shown as the mean of quadruplicate measurements \pm SD. ${ }^{* *} \mathrm{P}<0.01$ vs. control. (E) Scatterplot representation of the $c-M y c$ targeted genes validated by PCR array in HepG2 cells. siRNA, small interfering RNA; STEAP1, six-transmembrane epithelial antigen of the prostate 1; RT-qPCR, reverse transcription-quantitative PCR.

and NRF2 in three individual datasets (GSE14520, GSE36376 and TCGA; Fig. S4C-E). Furthermore, previous studies reported that c-Myc generates ROS in liver cancer cells $(24,25)$ However, the current study demonstrated that STEAP1 leads the increased expression of c-Myc and reduced ROS production in liver cancer cells. These results seem inconsistent, suggesting the existence of an NRF2 or c-Myc independent ROS-related pathway in the regulation of STEAP1-mediated cell growth. Additionally, others have shown STEAPI silencing induced cell growth inhibition, which was associated with decreased levels of ROS in cases of Ewing sarcoma (26). These results suggest the existence of multiple pathways between STEAP1 and ROS in a cancer-type specific manner. Accordingly, our next steps include exploring the relationship between STEAP1 and ROS in STEAP1-driven cancer cells.

In summary, this study provides a preclinical concept for STEAP1 as a druggable target in liver cancer, an often fatal cancer. The STEAP1-c-Myc axis has potential as an attractive and promising therapeutic target in liver cancer, and its manipulation will lead to the development of a novel strategy to conquer this malignant disease.

\section{Acknowledgements}

The authors would like to thank Ms. Kei Yoneguchi (Department of Medical Oncology, Sapporo Medical University School of Medicine, Sapporo, Hokkaido, Japan) for her technical assistance.

\section{Funding}

The present study was funded by a grant from Japan Society for the Promotion of Science (grant no. 19K08397)

\section{Availability of data and materials}

The datasets generated and/or analyzed during the current study are available in the Gene Expression Omnibus repository, https://www.ncbi.nlm.nih.gov/geo/query/acc. cgi?acc=GSE173813.

\section{Authors' contributions}

KT, HN and KI were responsible for the conception and design of the study and for confirming the authenticity of the data. NH, TK, YU, SI, KM, MK and JK performed the analysis and interpretation of data. HN and KT drafted the manuscript. JK critically reviewed and revised the manuscript. All authors read and approved the final manuscript.

\section{Ethics approval and consent to participate}

Not applicable.

\section{Patient consent for publication}

Not applicable. 


\section{Competing interests}

The authors declare that they have no competing interests.

\section{References}

1. Sung H, Ferlay J, Siegel RL, Laversanne M, Soerjomataram I Jemal A and Bray F: Global cancer statistics 2020: GLOBOCAN estimates of incidence and mortality worldwide for 36 cancers in 185 countries. CA Cancer J Clin 71: 209-249.

2. Llovet JM, Ricci S, Mazzaferro V, Hilgard P, Gane E, Blanc JF, de Oliveira AC, Santoro A, Raoul JL, Forner A, et al; SHARP Investigators Study Group: Sorafenib in advanced hepatocellular carcinoma. N Engl J Med 359: 378-390, 2008.

3. Bruix J, Qin S, Merle P, Granito A, Huang YH, Bodoky G, Pracht M, Yokosuka O, Rosmorduc O, Breder V, et al; RESORCE Investigators: Regorafenib for patients with hepatocellular carcinoma who progressed on sorafenib treatment (RESORCE): A randomised, double-blind, placebo-controlled, phase 3 trial. Lancet 389: 56-66, 2017.

4. Kudo M, Finn RS, Qin S, Han KH, Ikeda K, Piscaglia F, Baron A, Park JW, Han G, Jassem J, et al: Lenvatinib versus sorafenib in first-line treatment of patients with unresectable hepatocellular carcinoma: A randomised phase 3 non-inferiority trial. Lancet 391: 1163-1173, 2018.

5. Finn RS, Qin S, Ikeda M, Galle PR, Ducreux M, Kim TY, Kudo M, Breder V, Merle P, Kaseb AO, et al; IMbrave150 investigators: Atezolizumab plus bevacizumab in unresectable hepatocellular carcinoma. N Engl J Med 382: 1894-1905, 2020.

6. Stine ZE, Walton ZE, Altman BJ, Hsieh AL and Dang CV: MYC, metabolism, and cancer. Cancer Discov 5: 1024-1039, 2015.

7. Abou-Elella A, Gramlich T, Fritsch C and Gansler T: c-myc amplification in hepatocellular carcinoma predicts unfavorable prognosis. Mod Pathol 9: 95-98, 1996.

8. Schaub FX, Dhankani V, Berger AC, Trivedi M, Richardson AB Shaw R, Zhao W, Zhang X, Ventura A, Liu Y, et al; Cancer Genome Atlas Network: Pan-cancer alterations of the MYC oncogene and its proximal network across the Cancer Genome Atlas. Cell Syst 6: 282-300.e2, 2018.

9. Duffy MJ and Crown J: Drugging 'undruggable' genes for cancer treatment: Are we making progress? Int J Cancer 148: 8-17, 2021.

10. Massó-Vallés D and Soucek L: Blocking Myc to treat cancer: Reflecting on two decades of omomyc. Cells 9: 883, 2020.

11. Hubert RS, Vivanco I, Chen E, Rastegar S, Leong K, Mitchell SC, Madraswala R, Zhou Y, Kuo J, Raitano AB, et al: STEAP: A prostate-specific cell-surface antigen highly expressed in human prostate tumors. Proc Natl Acad Sci USA 96: 14523-14528, 1999.

12. Moreaux J, Kassambara A, Hose D and Klein B: STEAP1 is overexpressed in cancers: A promising therapeutic target. Biochem Biophys Res Commun 429: 148-155, 2012.

13. Gomes IM, Maia CJ and Santos CR: STEAP proteins: From structure to applications in cancer therapy. Mol Cancer Res 10: 573-587, 2012.

14. Oosterheert W and Gros P: Cryo-electron microscopy structure and potential enzymatic function of human six-transmembrane epithelial antigen of the prostate 1 (STEAP1). J Biol Chem 295: 9502-9512, 2020.
15. Nakamura H, Takada K, Arihara Y, Hayasaka N, Murase K, Iyama S, Kobune M, Miyanishi K and Kato J: Six-transmembrane epithelial antigen of the prostate 1 protects against increased oxidative stress via a nuclear erythroid 2-related factor pathway in colorectal cancer. Cancer Gene Ther 26: 313-322, 2019.

16. Menyhárt O, Nagy Á and Győrffy B: Determining consistent prognostic biomarkers of overall survival and vascular invasion in hepatocellular carcinoma. R Soc Open Sci 5: 181006, 2018.

17. Kanda Y: Investigation of the freely available easy-to-use software 'EZR' for medical statistics. Bone Marrow Transplant 48: 452-458, 2013.

18. Mani M, Carrasco DE, Zhang Y, Takada K, Gatt ME, Dutta-Simmons J, Ikeda H, Diaz-Griffero F, Pena-Cruz V, Bertagnolli M, et al: BCL9 promotes tumor progression by conferring enhanced proliferative, metastatic, and angiogenic properties to cancer cells. Cancer Res 69: 7577-7586, 2009.

19. Takada K, Zhu D, Bird GH, Sukhdeo K, Zhao JJ, Mani M, Lemieux M, Carrasco DE, Ryan J, Horst D, et al: Targeted disruption of the BCL9/ $\beta$-catenin complex inhibits oncogenic Wnt signaling. Sci Transl Med 4: 148ra117, 2012.

20. Hayasaka N, Takada K, Nakamura H, Arihara Y, Kawano Y, Osuga T, Murase K, Kikuchi S, Iyama S, Emori M, et al: Combination of eribulin plus AKT inhibitor evokes synergistic cytotoxicity in soft tissue sarcoma cells. Sci Rep 9: 5759, 2019.

21. Matsuoka K, Koreth J, Kim HT, Bascug G, McDonough S, Kawano Y, Murase K, Cutler C, Ho VT, Alyea EP, et al: Low-dose interleukin-2 therapy restores regulatory $\mathrm{T}$ cell homeostasis in patients with chronic graft-versus-host disease. Sci Transl Med 5: 179ra43, 2013.

22. Llovet JM, Montal R, Sia D and Finn RS: Molecular therapies and precision medicine for hepatocellular carcinoma. Nat Rev Clin Oncol 15: 599-616, 2018.

23. Danila DC, Szmulewitz RZ, Vaishampayan U, Higano CS, Baron AD, Gilbert HN, Brunstein F, Milojic-Blair M, Wang B, Kabbarah O, et al: Phase I study of DSTP3086S, an antibody-drug conjugate targeting six-transmembrane epithelial antigen of prostate 1 , in metastatic castration-resistant prostate cancer. J Clin Oncol 37: 3518-3527, 2019.

24. Dolezal JM, Wang H, Kulkarni S, Jackson L, Lu J, Ranganathan S, Goetzman ES, Bharathi SS, Beezhold K, Byersdorfer CA, et al: Sequential adaptive changes in a c-Myc-driven model of hepatocellular carcinoma. J Biol Chem 292: 10068-10086, 2017.

25. Zheng K, Cubero FJ and Nevzorova YA: c-MYC-making liver sick: Role of c-MYC in hepatic cell function, homeostasis and disease. Genes (Basel) 8: 123, 2017.

26. Grunewald TG, Diebold I, Esposito I, Plehm S, Hauer K, Thiel U, da Silva-Buttkus P, Neff F, Unland R, Müller-Tidow C, et al: STEAP1 is associated with the invasive and oxidative stress phenotype of Ewing tumors. Mol Cancer Res 10: 52-65, 2012.

This work is licensed under a Creative Commons Attribution-NonCommercial-NoDerivatives 4.0 International (CC BY-NC-ND 4.0) License. 